\title{
GW23-e2621 EFFECTS OF HIGH SALT DIET ON ARTERIAL REMODELLING AND THE INTERVENTION OF TELMISARTAN IN WISTAR RATS
}

doi:10.1136/heartjnl-2012-302920a.199

${ }^{1}$ Oian-Hui Shang, ${ }^{1}$ Xiao-Oiang Min, ${ }^{1}$ Chan Liu, ${ }^{1}$ Wan-Heng Mao, ${ }^{2}$ Oian-Hui Shang. ${ }^{1}$ Institute of Clinical Medicine, Department of cardiology of Affiliated Hospital, Zunyi Medical College; ${ }^{2}$ Institute of Clinical Medicine, Department of cardiology of Affiliated Hospital, Zunyi

Objectives To investigate the effects of dietary salt on aorta and mesenteric artery remodelling in Wistar Rats and explore the possible mechanism of salt-induced arterial remodelling and AngiotensinII receptor blockers telmisartan intervention.

Methods 60 Wistar rats were randomly divided into normal control group, high salt (8\%) model group and high salt+telmisartan group. The tail artery pressure was determined every 2 weeks. After 24 weeks, high salt model group was divided into model hypertension $(\mathrm{MH})$ group and model normal pressure $(\mathrm{MN})$ group. The structural changes and proliferation in the media of aorta and mesenteric arteries were observed by HE staining, Masson staining and immunohistochemical staining. The activities and mRNA levels of $\mathrm{Na}^{+}$pump and $\mathrm{Ca}^{2+}$ pump in aortic media were determined by enzyme colorimetry and real-time PCR respectively.

Results Compared with control group, the blood pressure was significantly increased in $\mathrm{MH}$ Group, Media thickness (MT), lumen diameter (LD), ratio of media to lumen (MT/LD), the collagen volume fraction and PCNA positive expressive percentage of arteries in high-salt group were increased $(p<0.05)$, the activities of $\mathrm{Na}^{+}-\mathrm{K}^{+}$-ATPase and $\mathrm{Ca}^{2+}$-ATPase in $\mathrm{MH}$ group were decreased $(p<0.05)$.The mRNA expression of $\mathrm{Na}^{+}-\mathrm{K}^{+}$-ATPase $\alpha_{1}$ subunit in 
$\mathrm{MH}$ and $\mathrm{MN}$ groups was decreased $(\mathrm{P}<0.05)$, and PMCA1 expression raised in $\mathrm{MH}$ group, Correlation analysis showed that two ATPase activities and vascular remodelling indicators have a negative correlation $(p<0.05)$.Compared with high-salt group, blood pressure, media thickness, ratio of media to lumen, the collagen volume and PCNA positive expressive percentage were lower in telmisartan group $(p<0.05)$.

Conclusions High-salt diet could lead to arterial remodelling directly or indirectly (elevated blood pressure), The decreased ion pump activity and abnormal gene expression may be one of the mechanisms of high-salt induced arterial remodelling. Telmisartan may inhibit the proliferation of vascular smooth muscle and collagen accumulation, and prevent salt-induced hypertension and arterial remodelling. 